controversial issue. Our study aims to investigate three different methods of quantifying Ki-67 in both pulmonary and mediastinal NETs. We retrieved 40 cases of pulmonary and mediastinal NETs. Manual counting with a graticule, manual counting on a printed image of the hotspot area and automated digital image analysis (DIA) were used to calculate the $\mathrm{Ki}-67$ proliferative index.

\section{METASTATIC PLEOMORPHIC DERMAL SARCOMA TO SMALL BOWEL - A CASE REPORT}

\author{
Joanna Kang, Eileen Long \\ Anatomical Pathology Department, Hobart Pathology, Hobart, \\ Australia
}

Pleomorphic dermal sarcoma (PDS), a dermal based tumour that is related to atypical fibroxanthoma with overlapping morphological and immunohistochemical features but with subcutaneous invasion, necrosis, perineural or vascular invasion. PDS has low malignant potential with risk of local recurrence and metastases. Reported sites of metastases are distant skin, lymph nodes and lung. We report a case of a male aged 65 who presented with gastrointestinal bleeding due to haemorrhage from a small bowel lesion. His medical history included MuirTorre syndrome, stage IV colon cancer (with liver metastases) and PDS of the lower lip (resected February 2018) with known lung metastases. He underwent a segmental resection of small bowel in June 2019; macroscopically a solitary $39 \mathrm{~mm}$ lesion was noted. Histopathology showed a tumour composed of sheets and nests of pleomorphic, mitotically active, epithelioid and spindle cells. Immunohistochemically the malignant cells showed positive for CD10 and negative staining for cytokeratins, CD117 and melanoma markers. The morphology and immunophenotype were the same as his previously diagnosed lip PDS. This is the first reported case of metastatic PDS to small bowel, and highlights the importance of clinical-pathological correlation. Although rare, metastatic sarcoma should be considered in the differential of pleomorphic epithelioid/spindled small bowel neoplasms.

\section{References}

1. Tardío JC, Pinedo F, Aramburu JA, et al. Pleomorphic dermal sarcoma: a more aggressive neoplasm than previously estimated. $J$ Cutan Pathol 2016; 43: 101-12.

2. Miller K, Goodlad JR, Brenn T. Pleomorphic dermal sarcoma adverse histologic features predict aggressive behavior and allow distinction from atypical fibroxanthoma. Am J Surg Pathol 2012; 36: 1317-26.

\section{AN UNUSUAL CASE OF METASTATIC GASTRIC ADENOCARCINOMA TO THE OVARY WITH YOLK SAC DIFFERENTIATION AND AFP PRODUCTION}

A. Kang ${ }^{1,2}$, L. Kang ${ }^{2}$, C. J. R. Stewart ${ }^{1}$, M. H. Eleanor Koay ${ }^{1,2}$ ${ }^{1}$ Department of Anatomical Pathology, King Edward Memorial Hospital, WA, Australia; and ${ }^{2}$ Department of Anatomical Pathology, Fiona Stanley Hospital, Murdoch, WA, Australia

Raised serum AFP in adults is most commonly associated with hepatocellular carcinoma (HCC) or yolk sac tumour (YST). However, studies have reported diseases other than HCC or YST which are associated with high serum AFP levels. We describe a case of metastatic adenocarcinoma to the ovary showing yolk sac differentiation, as a primary presentation of a gastric tumour.

\section{AUDIT ON THE PERFORMANCE OF MMR PROTEIN IHC STAINING ON BIOPSIES AND RESECTIONS OF COLORECTAL CANCER}

L. Kang ${ }^{1}$, W. B. de Boer ${ }^{2}$

${ }^{I}$ School of Medicine and Public Health, University of Newcastle, Newcastle, NSW, Australia; and ${ }^{2}$ Department of Anatomical Pathology, PathWest Fiona Stanley Hospital, Murdoch, WA, Australia

Colorectal cancer may arise from an accumulation of uncontrolled DNA mutations that is a result of damage to or absence of mismatch repair (MMR) genes. These DNA damage repair genes consist primarily of MLH1, PMS2, MSH2 and MSH6. Mismatch repair deficient (MMRD) colorectal cancers may arise from acquired or inherited loss of protein expression; the latter referred to as Lynch syndrome.

Immunohistochemical (IHC) testing for MMR protein expression is part of the standard of care for the work up of colorectal cancer. Identification of absent protein expression prompts referral of the patient to genetic services for further work up. The aim of our study was to audit the performance of MMR protein IHC staining on biopsies and resections of colorectal cancer, in particular turnaround-time. This is relevant to patient review at multidisciplinary team meetings and timely referral to genetic services when appropriate.

\section{UTILITY OF KI67 AND P53 IN EARLY DETECTION AND PROGNOSIS ASSESSMENT OF ORAL SQUAMOUS CELL CARCINOMA}

Saurabh N. Tripathi, Neelkamal Kapoor, Garima Goel, Deepti Joshi

Department of Pathology and Lab Medicine, All India Institute of Medical Sciences Bhopal, India

Carcinoma of the oral cavity constitutes $3 \%$ of all cancers. The incidence is higher in India because of use of smokeless tobacco (chewing). $90 \%$ of oral cavity carcinomas are oral squamous cell carcinoma (OSCC) and mucosa surrounding them more than often shows features of dysplasia of varying degree. It is well established fact that early detection and timely intervention in case of oral cancer reduces the morbidity, mortality and cost of treatment. Hence it is important to explore the use of ancillary techniques for early detection and assessment of prognosis. Molecular proliferation markers Ki-67 and p53 can be used for assessing the potential of malignancy in the tumour tissue and its surrounding dysplastic tissue. In the present study tissue sections from 64 paraffin blocks containing tissue from cases of OSCC and surrounding dysplastic area were immunohistochemically stained for $\mathrm{Ki}-67$ and p53. It was found that for $\mathrm{Ki}-67$ among the tumour area 58 cases were positive and 32 cases showed positivity in dysplastic areas. For p53 the positivity was 23 and 9 for the tumour and dysplastic areas, respectively. Significant $p$ value was obtained to indicate usefulness of utilising these markers for early detection and prognostication. 